\title{
L'ACTINE CYTOSQUELETTIQUe ET SES PROTÉINES ASSOCIÉES QUELQUES EXEMPLES CHEZ LES PROTISTES
}

\author{
GUILLÉN N.*, CARLIER M.-F.**, BRUGEROLLE G.***, TARDIEUX I.**** \& AUSSEIL J.*****
}

\section{Résumé :}

La mise en oeuvre de multiples processus biologiques nécessite des changements du cytosquelette riche en actine. Tel est le cas de la motilité cellulaire qui résulte de l'assemblage et désassemblage des filaments d'actine, une activité régulée par l'action de nombreuses protéines liant l'actine. Les organismes unicellulaires sont des modèles de choix pour l'étude des régulations engendrant les remaniements du cytosquelette car leur manipulation aisée s'adapte à diverses technologies. Lors de la XXXVe réunion annuelle du Groupement des Protistologues de Langue Française (GPLF), un symposium concernant « L'actine cytosquelettique et ses protéines associées chez les protistes " a été organisé. Ce colloque reflète l'intérêt du GPLF pour le développement en France des thèmes de recherche concernant des protistes pathogènes pour l'homme et ceux, pathogènes ou non, servant de modèles d'analyse cellulaire. Un compte rendu de ce colloque est présenté dans cet article.

MOTS CLÉS : actine, cytosquelette, protistes.
Summary : ACTIN CYTOSKELETON AND ASSOCIATED PROTEINS: A FEW EXAMPLES IN PROTISTS

Many processes, cell motility being an example, require cells to remodel the actin cytoskeleton in response to both intracellular and extracellular signals. Reorganization of the actin cytoskeleton involves the rapid disassembly and reassembly of actin filaments, a phenomenon regulated by the action of particular actin-binding proteins. In recent years, an interest in studying actin regulation in unicellular organisms has arisen. Parasitic protozoan are among these organisms and studies of the cytoskeleton functions of these protozoan are relevant related to either cell biology or pathogenicity. To discuss recent data in this field, a symposium concerning "Actin and actin-binding proteins in protists " was held on May 8-1 1 in Paris, France, during the XXXV meeting of the French Society of Protistology.

As a brief summary of the symposium we report here findings concerning the in vitro actin dynamic assembly, as well as the characterization of several actin-binding proteins from the parasitic protozoan Entamoeba histolytica, Thrichomonas vaginalis and Plasmodium knowlesi. In addition, localization of actin in nonpathogen protists such as Prorocentrum micans and Crypthecodinium cohnii is also presented.

The data show that some actin-binding proteins facilitate organization of filaments into higher order structures as pseudopods, while others have regulatory functions, indicating very particular roles for actin-binding proteins. One of the proteins discussed during the symposium, the actin depolymerizing factor ADF, was shown to enhance the treadmilling rate of actin filaments. In vitro, ADF binds to the ADP-bound forms of G-actin and F-actin, thereby participating in and changing the rate of actin assembly. Biochemical approaches allowed the identification of a protein complex formed by HSP/C70-cap32-34 which might also be involved in depolymerization of $F$-actin in $P$. knowlesi. Molecular and cellular approaches were used to identify proteins such as ABP-120 and myosin $\mathrm{B}$ at the leading edge of E. histolytica. ABP-1 20 organizes F-actin in a network and myosin IB participates in the pseudopod formation. Similar approaches using $T$. vaginalis resulted in the discovery of an actin-binding protein that participate in the F-actin reorganization during adhesion of parasites to target cells. This protein is homologous to $\alpha$-actinin from other eukaryotic cells. Finally, by using cell biology approaches, F-actin was observed in the cytoplasm as well as in the nucleus of Dinoflagelates. The recent developments in the molecular genetics of protozoa will provide new insights to understand the roles of actin-binding proteins during cytoskeleton activities.

KEY WORDS : actin, cytoskeleton, protists. 


\section{INTRODUCTION}

$\Gamma$ 'actine est l'un des constituants majeurs de toute cellule eucaryote et apparait comme l'une des protéines la mieux conservée au cours de l'évolution. Son importance est bien connue dans la structure et la fonction des cellules musculaires mais elle l'est moins dans les autres cellules où cette protéine constitue un des éléments du cytosquelette et intervient dans des fonctions très variées. La compréhension des phénomènes de motilité cellulaire, de morphogénèse, de certains mouvements intracellulaires, d'adhésion et de signalisation, voire de cytodiérèse nécessite de connaître les propriétés de l'actine et des protéines qui lui sont associées.

L'activité biologique de l'actine est régulée par son état physique et par sa liaison à de nombreuses protéines affines. En effet, l'actine a une forte tendance à s'assembler en filaments hélicoïdaux (actine filamenteuse ou F-actine), mais cet état est en équilibre avec l'actine monomérique (G-actine). Le maintien physiologique de cet équilibre dépend des protéines capables de séquestrer les monomères d'actine et de désagréger les filaments. La polymérisation de l'actine n'a pas lieu uniformément dans le cytoplasme mais dans des sites discrets dénommés sites de nucléation en général localisés aux alentours de la membrane plasmique. Des questions importantes sont donc posées pour comprendre l'équilibre entre F-actine et G-actine et pour déterminer, d'une part, la régulation moléculaire qui associe les microfilaments d'actine avec d'autres protéines et d'autre part, le mécanisme qui régule aussi bien la morphologie que le mouvement des cellules. Une synthèse des connaissances actuelles sur la polymérisation de l'actine et le rôle des protéines liant l'actine seront d'abord présentés : ces résultats ont été obtenus par des études in vitro mais ils peuvent être transposés au cytoplasme de la cellule vivante. Les protistes, ou premiers eucaryotes, sont très divers et offrent de nombreux modèles où les multiples fonctions de l'actine peuvent être étudiées. Le cytosquelette des protistes contient diverses structures filamenteuses associées à des fonctions précises. L'intervention de l'actine dans la biologie de protistes sera révélée dans quatre exemples qui font l'objet de recherches actuelles dans des équipes françaises. L'amibe pathogène Entamoeba bistolytica est capable de mouvements amiboïdes et de phagocytose, phénomènes qui font intervenir le cytosquelette d'actine. Le flagellé parasite Trichomonas vaginalis devient amiboïde en adhérant aux cellules de l'hôte et développe un réseau d'actine périphérique. La motilité des Sporozoaires Plasmodium et Toxoplasma et leur pénétration dans la cellule hôte semble bien dépendre du cytosquelette d'actine. Les Dinoflagellés sont les seuls organismes à présenter des fibres d'actine dans leur noyau, la division de leur appareil flagellaire et leur morphogénèse font aussi intervenir le cytosquelette d'actine.

\section{POLYMÉRISATION DE L'ACTINE ET MOTILITÉ CELLULAIRE}

I 1 est bien admis que les mouvements de locomotion des cellules vivantes, et plus généralement leurs changements de forme en réponse aux stimuli extracellulaires, sont engendrés par la polymérisation de l'actine (Wang, 1985; Stossel, 1993). La polymérisation des filaments d'actine peut développer en effet une force de protrusion capable de déformer la membrane plasmique (Mogilner et Oster, 1996).

La motilité cellulaire liée à la polymérisation de l'actine est caractérisée par deux aspects essentiels; d'une part la capacité d'une cellule à augmenter rapidement la quantité d'actine polymérisée en réponse à un signal. Par exemple, l'activation des plaquettes sanguines, des leucocytes polymorphonucléaires ou la stimulation de l'amibe Dictyostelium discoideum par un agent chimiotactique sont suivies immédiatement (temps de réponse : $10 \mathrm{~s}$ ) par une polymérisation massive de filaments. L'augmentation de la concentration d'actine polymérisée dans la cellule se fait aux dépens du réservoir d'actine non polymérisée, maintenue monomérique par interaction avec des protéines qui l'empêchent de polymériser, connues sous le nom de "protéines séquestrant l'actine ", telles la thymosine $\beta_{4}$ et la profiline. Le mécanisme de contrôle du passage de l'actine de la forme séquestrée à la forme polymérisée est un élément crucial de la motilité cellulaire.

Un deuxième phénomène fascinant qui caractérise la motilité cellulaire est l'usage fait par la cellule du renouvellement (" turnover ") des filaments d'actine pour générer le mouvement. L'assemblage continu des filaments en un réseau ponté, au front de migration des cellules, constitue la force de protrusion du cytoplasme qui permet l'extension du lamellipode. Cet assemblage localisé de filaments est compensé par la dépolymérisation des filaments à l'arrière du lamellipode, créant un phénomène de "tapis roulant " ou " treadmilling ". La vitesse du mouvement (1$10 \mu \mathrm{m} / \mathrm{min}$ ) implique que la vitesse d'allongement des filaments d'actine est de 10 à 100 sous-unités par seconde. Dans un tel régime stationnaire, la quantité d'actine polymérisée reste constante, et la question cruciale est de comprendre " comment une cellule peut maintenir des vitesses de polymérisation et de dépolymérisation aussi élevées simultanément en différentes régions du cytoplasme "(Forscher and Smith, 1988; Fechheimer et Zigmond, 1993). 


\section{L'ÉTAT STATIONNAIRE DE POLYMÉRISATION} DE L'ACTINE

Dans le milieu cellulaire qui est riche en ATP-Mg et de force ionique élevée, l'actine est polymérisée à l'état stationnaire, c'est-à-dire que la masse d'actine polymérisée est constante, et les filaments coexistent avec l'actine monomérique (G-ATP) maintenue elle aussi à une concentration constante par le jeu des échanges dynamiques monomère-polymère dans le milieu. Cette concentration stationnaire d'actine monomérique n'est pas une concentration critique à proprement parler, c'est-à-dire une constante d'équilibre monomère-polymère. En effet, comme l'hydrolyse irréversible de l'ATP accompagne la polymérisation de l'actine G-ATP en filaments, le polymère et le monomère sont dans un état stationnaire, et il existe un flux des sous-unités d'actine à travers le polymère, appelé " treadmilling ", qui assure le turnover des filaments et du réservoir des sous-unités d'actine monomérique. Dans le processus de treadmilling, la vitesse nette de dépolymérisation à l'extrémité pointue des filaments est égale à la vitesse nette de polymérisation à l'extrémité barbée. Ceci signifie que la concentration stationnaire d'actine GATP est inférieure à la concentration critique d'assemblage à l'extrémité pointue et supérieure à la concentration critique d'assemblage à l'extrémité barbée.

Ces caractéristiques fondamentales de la polymérisation de l'actine sont utilisées par la cellule vivante et modulées par des protéines associées à l'actine pour contrôler les processus de séquestration/déséquestration de l'actine, et réguler le turnover des filaments.

\section{CONTRÔLE DE LA SÉQUESTRATION/DÉSÉQUESTRATION DE L'ACTINE MONOMÉRIQUE}

Au cours des 10 dernières années, le rôle des protéines dites de séquestration de l'actine monomérique, thymosine $\beta_{4}$ et ses variants $\left(\beta_{9}, \beta_{10}, \beta_{15}, \ldots\right)$ a été précisé. Ces protéines fixent spécifiquement l'actine G-ATP et l'empêchent de polymériser. Elles sont en équilibre relativement rapide avec l'actine, par conséquent, la concentration de complexe G-actine-thymosine est régie par la loi d'action de masse. La concentration d'actine monomérique G-ATP étant maintenue stationnaire $\left(\mathrm{C}_{\mathrm{ss}}\right)$, la concentration de complexe l'est également. Il s'en suit que la seule façon de faire varier la concentration d'actine séquestrée est d'agir sur la concentration stationnaire d'actine G-ATP. Les protéines régulatrices qui possèdent cette fonction sont les protéines de coiffe, ou " capping proteins ", qui augmentent $C_{s s}$ jusqu'à la valeur de la concentration critique d'assemblage à l'extrémité pointue, et la profiline, qui possède la propriété inverse d'abaisser la valeur de la concentration critique d'assemblage à l'extrémité barbée (Pantaloni et Carlier, 1993). Les variations de $\mathrm{C}_{\mathrm{ss}}$ sont accompagnées de variations de même sens de la concentration d'actine séquestrée, mais ces variations sont amplifiées d'une manière proportionnelle à la concentration totale de protéine séquestrante. Ainsi les " capping proteins ", en maintenant une valeur élevée de $\mathrm{C}_{\mathrm{ss}}$, établissent un large réservoir d'actine non polymérisée (c'est la situation des cellules motiles dans l'état quiescent). La profiline au contraire, en abaissant $\mathrm{C}_{\mathrm{s}}$, fait diminuer la concentration d'actine monomérique séquestrée, donc promeut une polymérisation massive d'actine. Cette fonction de la profiline est essentielle dans de nombreuses étapes du développement précoce où la polymérisation massive de l'actine est en jeu. L'absence de profiline est léthale chez tous les organismes pluricellulaires. L'abaissement de la concentration d'actine G-ATP stationnaire par la profiline est une conséquence directe de l'assemblage des filaments à l'extrémité barbée à partir de complexe profiline-actine, et n'est pas dû à l'augmentation de la vitesse d'échange du nucléotide sur l'actine monomérique par la profiline (Perelroizen et al., 1996). Le complexe profiline-actine peut, à ce titre, être considéré comme une autre espèce polymérisable d'actine.

\section{RÉGULATION DU TURNOVER DES FILAMENTS D'ACTINE PAR L'ADF/COFILINE}

La cofiline ou "actin depolymerizing factor "(ADF) est une petite protéine associée à l'actine, ubiquitaire, et dont l'absence cause des déficiences graves dans la translocation du centrosome et la cytocinèse, conduisant à la mort cellulaire (Abe et al., 1996). La surexpression de l'ADF en revanche augmente la motilité chez Dictyostelium. L'ADF a été d'abord considéré comme une protéine séquestrant l'actine monomérique comme la thymosine $\boldsymbol{\beta}_{4}$, et capable de fragmenter les filaments. Ces notions ont été remises en question récemment (Carlier et al., 1997) par une étude biochimique in vitro. Nous avons en effet montré que l'ADF, en interagissant à la fois avec l'actine G-ADP et l'actine F-ADP, participait au cycle d'assemblage stationnaire de l'actine en modifiant les paramètres cinétiques. L'effet majeur de l'ADF est d'augmenter de plus d'un ordre de grandeur la vitesse de dépolymérisation des filaments à l'extrémité pointue, qui est l'étape cinétiquement limitante du treadmilling. Le turnover des filaments in vitro en présence d'ADF devient alors comparable à celui qui est observé dans les cellules vivantes. L'augmentation de vitesse de treadmilling dû à l'ADF a des conséquences importantes pour la motilité. Elle implique en effet une augmentation de la concentration stationnaire d'actine G-ATP, et de la vitesse stationnaire d'assemblage de l'actine à l'extrémité barbée. 
La fixation de l'ADF aux filaments d'actine cause un profond changement de structure des filaments, qui se manifeste par une diminution importante de la viscosité des solutions de F-actine. Il est possible que l'ADF soit ainsi responsable de la transition gel-sol qui se produit en réponse à différents stimuli extracellulaires. L'activité de l'ADF comme stimulateur de la dynamique des filaments d'actine est régulée par phosphorylation réversible de la sérine 3. L'ADF déphosphorylé est actif. La déphosphorylation de l'ADF se produit en liaison avec les processus de signalisation cellulaire impliqués dans la croissance et l'endocytose. La kinase et la phosphatase responsables de la phosphorylation/déphosphorylation de l'ADF ne sont pas encore identifiées.

En conclusion, l'analyse du rôle de différentes protéines associées à l'actine dans la régulation de la dynamique des filaments indique que le contrôle de l'état stationnaire d'assemblage des filaments joue un rôle clé dans la motilité. Les protéines régulatrices de la polymérisation pourraient agir de façon synergique dans ce contrôle. Les questions qui restent ouvertes concernent le mécanisme de nucléation des filaments en des sites privilégiés de la membrane plasmique, et la nature des protéines (et leurs interactions) qui assurent la connexion entre le réseau d'actine et les voies de signalisation.

\section{PROTÉINES DU CYTOSQUELETTE NÉCESSAIRES À LA FORMATION DU FRONT DE MIGRATION CHEZ ENTAMOEBA HISTOLYTICA}

ntamoeba histolytica, protozoaire parasite de
l'homme, est responsable de l'amibiase intesti-
nale aiguë. Cette maladie, qui atteint $10 \%$ de la population mondiale, est caractérisée par une dysenterie et dans $10 \%$ des cas par le développement d'abcès du foie. La contamination se fait par l'intermédiaire de la forme enkystée du parasite qui est transmise par l'eau et l'alimentation. C'est la forme trophozoïte de E. bistolytica qui est responsable de l'amibiase. En présence de certains stimuli environnementaux encore inconnus, les trophozoïtes résidant dans l'intestin deviennent virulents, lysent et phagocytent les cellules épithéliales, puis gagnent la circulation sanguine et colonisent en particulier le foie où ils forment des abcès.

De multiples changements morphologiques ont lieu chez E. bistolytica lors de la phagocytose des cellules humaines et durant le mouvement à travers l'épithélium intestinal (Guillén, 1996). Par exemple le déploiement des pseudopodes est nécessaire à sa mobilité et à ses activités phagocytaires. L'extension d'un pseudopode est la base de la locomotion des cellules de type amiboïde, elle se produit par l'interaction des filaments d'actine avec d'autre protéines. L'identification de protéines participant avec l'actine à la formation du pseudopode est un des objectifs majeurs dans l'étude du cytosquelette amibien.

Pour purifier l'actine, en essayant de conserver son interaction avec les protéines qui lui sont associées, nous avons précipité des complexes protéiques à haute concentration de sels, puis ceux-ci ont été soumis à une chromatographie sur gel de sépharose $4 \mathrm{~B}$. Nous avons ainsi isolé un complexe protéique composé d'actine et de plusieurs protéines, parmi lesquelles a été identifié le facteur de gélation ABP-120 (Vargas, 1996). Cette protéine, déjà décrite dans une amibe nonpathogène, Dictyostelium discoideum, organise l'actine en réseau dans la région du pseudopode (Noegel et al., 1995). Nous avons isolé chez E. histolytica le gène codant cette protéine et sa séquence nucléotidique a été déterminée. L’analyse de la séquence protéique déduite de l'information nucléotidique a montré que la protéine ABP-120 de E. histolytica (EhABP-120) présente $54 \%$ de similitude avec la protéine ABP-120 de $D$. discoideum et avec la protéine ABP-280 humaine (filamine). De plus, la comparaison des domaines de liaison à l'actine en position amino-terminale montre que la similitude est de $67 \%$ entre E. histolytica et $D$. discoideum et de $60 \%$ entre $E$. histolytica et la protéine humaine. Ces homologies suggèrent des fonctions équivalentes pour ces trois protéines. En effet, le domaine amino-terminal de EhABP-120 lie l'actine dans des expériences in vitro. Par ailleurs EhABP-120 possède dans sa région carboxy-terminale un bloc de 95 amino acides non retrouvé dans les protéines homologues décrites. Ce domaine présente une forte homologie avec les protéines qui lient les microtubules dans les cellules eucaryotes.

Afin de localiser EhABP-120 dans les amibes en mouvement, un anticorps qui reconnaît spécifiquement le domaine amino-terminal de la protéine a été produit. Des trophozoïtes de $E$. bistolytica ont été fixés, marqués avec cet anticorps et examinés en immunofluorescence par microscopie confocale à rayon laser. Nous avons montré que l'ABP-120 dans les amibes en mouvement est effectivement localisée dans le pseudopode (fig. 1).

Deux autres protéines nécessaires à la formation du pseudopode chez E. histolytica ont été identifiées : la profiline ( $N$. Guillén et $M$. Duchêne, résultats non publiés), et la myosine IB (Vargas, 1997). La première activité décrite pour la profiline est celle d'induire la dépolymérisation de l'actine par sa liaison avec la forme G-actine monomérique. Cependant, des travaux plus récents ont montré que la profiline a d'autres fonc- 


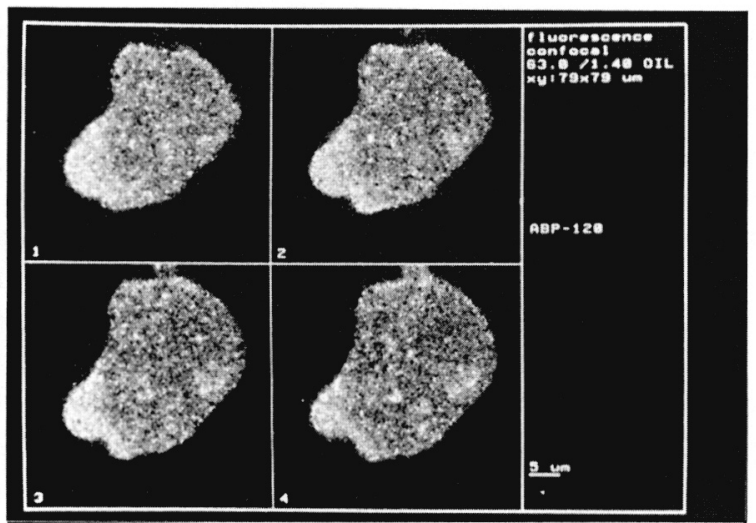

B

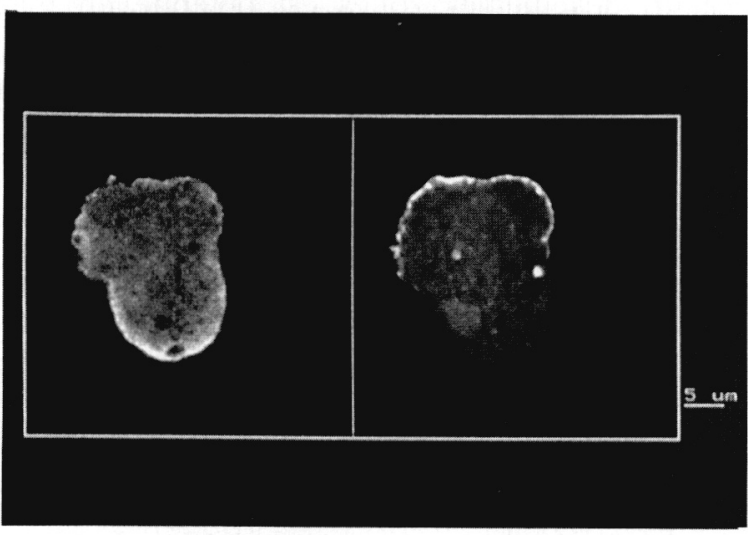

Fig. 1. - Distribution d'ABP-120 et de la Myosine IB chez Entamoeba histolytica en mouvement. A. Distribution d'ABP-120. Les amibes en croissance végétative ont été fixées au paraformaldehyde 3,7\%, perméabilisées au Triton X-100 0,1\% et incubées en présence d'un anticorps anti-EhABP120 puis révélées avec un anti IgG de lapin couplé à la rhodamine. L'immunofluorescence est analysée par microscopie confocale à rayon laser. Quatre sections optiques $(0,5 \mu \mathrm{m})$ à différents plans de la cellule sont montrées. EhABP120 se retrouve dispersée dans le cytoplasme et concentrée dans la région du pseudopode.

B. Distribution de la myosine IB. Préalablement les amibes sont incubées en présence de Concanavaline A couplée au FITC. Après traitement comme indiqué en $\mathrm{A}$, les amibes sont colorées par un anticorps anti-myosine IB de rat. Dans le panel de gauche la distribution de la myosine IB est montrée, cette protéine se retrouve près de la membrane plasmique et plus concentrée dans le pseudopode. Dans la partie droite de la micrographie la partie arrière de la cellule est visualisée par concentration de récepteurs chargés à la ConA-FITC.

tions. En effet, elle est capable de promouvoir l'échange de la molécule d'ADP par de l'ATP lié à l'actine; cette protéine peut également diminuer la concentration critique de l'ATP-actine. Dans ces deux conditions particulières la profiline engendre la polymérisation de l'actine. Au vu de ces fonctions il est à espérer que la profiline joue un rôle régulateur de la formation du pseudopode chez E. histolytica.

La myosine IB est une mécanoenzyme qui confère l'énergie nécessaire au déplacement des microfila- ments d'actine. La myosine IB de E. histolytica est formée de 1049 acides aminés (Vargas, 1997). Elle possède une chaîne lourde comportant des domaines spécifiques définis comme : la tête, le cou et la queue. La tête est l'unité motrice qui contient les sites de liaison à l'actine et à l'ATP. La queue se divise en trois sous-domaines : le domaine d'interaction avec des membranes, un domaine atypique de liaison à l'actine, ATP indépendant, et un troisième domaine $\mathrm{SH} 3$ important pour l'interaction avec d'autres protéines. Enfin, un motif IQ est présent dans le cou, il est éventuellement impliqué dans l'association des chaînes légères. Nous avons étudié la capacité à lier l'actine du site ATP-indépendant. En effet, la protéine identifiée est homologue à la myosine IB des autres amibes mais une différence a été cependant rencontrée car le deuxième domaine de liaison à l'actine ne comporte que 49 amino acides, alors que dans les autres myosines IB ce domaine est de 170 amino acides. En exprimant chez Escherichia coli le domaine carboxyterminal de la myosine identifiée, et par des expériences d'interaction de ce domaine avec des extraits d'amibes, nous avons montré qu'il est suffisant pour lier l'actine (Vargas, 1997). Enfin, en utilisant des anticorps hétérologues dirigés contre la protéine de rat, la myosine IB a pu être localisée dans le pseudopode (fig. 1), suggérant pour cette protéine un rôle dans la locomotion de E. histolytica.

En résumé, trois protéines candidates pour l'étude du mécanisme de formation de pseudopodes chez le parasite E. histolytica ont été identifiées : le facteur de gélation ABP-120, la profiline et la myosine IB. Il est important de comprendre la part qui revient à chacune de ces protéines dans cette fonction. Une hypothèse de travail est que la profiline servirait de régulateur de la polymérisation de l'actine dans le pseudopode, alors que l'ABP-120 aurait le rôle de structurer cet appendice. Par ailleurs, la myosine IB aurait la charge de produire l'énergie nécessaire pour déplacer le réseau d'actine en interaction avec la membrane plasmique.

\section{L'ACTINE ET LES PROTÉINES QUI LUI SONT ASSOCIÉES CHEZ LE PROTOZOAIRE PARASITE TRICHOMONAS VAGINALIS}

\footnotetext{
$\mathrm{E}$ n général tous les flagellés du groupe des Trichomonadines et des Parabasala se nourrissent par phagocytose et pinocytose, et certains adoptent une morphologie amiboïde bien qu'ils ne se déplacent généralement pas à l'aide de mouvements amoeboïdes. Les espèces de Trichomonadines capables d'envahir les tissus comme Histomonas meleagridis et
} 
Trichomonas gallinae (parasites des coecums et du foie des oiseaux), Trichomonas vaginalis (parasite du tractus uro-génital de l'homme) et Dientamoeba fragilis (parasite intestinal de l'homme), sont celles chez qui l'amoeboïsme est le plus développé.

Il est connu que Trichomonas vaginalis est capable d'adhérer aux cellules épithéliales et de prendre une forme amiboïde chez l'hôte naturel ou lorsqu'il est injecté à des souris (Alderete et al., 1995; Brugerolle et al., 1996). Dans un modèle in vitro, T. vaginalis a pu être cultivé sur lamelles. Les flagellés ovoïdes se transforment en cellules adhérentes amiboïdes et forment une monocouche (fig. 2a et 2b). Ces cellules présentent une frange périphérique de cytoplasme homogène riche en microfilaments semblables à ceux qui sont révélés lorsque $T$. vaginalis adhère aux cellules des tissus de l'hôte (fig. 2d et 2e) (Brugerolle et al., 1996).
La présence d'actine avait été démontrée chez T. vaginalis (Cappuccinelli et al., 1987) où elle est la protéine majoritaire $(13,4 \%)$. Une étude électrophorétique et immunologique montre que cette protéine a une masse moléculaire de $42 \mathrm{kDa}$ comme l'actine musculaire. En électrophorèse bidimensionnelle, elle se résout en au moins quatre spots dont le majeur a un pI de 5,4, laissant supposer qu'il existe plusieurs isoformes. L'étude en immunofluorescence (IF) en utilisant la phalloïdineTRITC ou des anticorps (Ac) monoclonaux anti-actine révèle l'actine dans tout le cytoplasme de la forme flagellée et dans la forme adhérente amiboïde (fig. 2c). Dans cette dernière, la frange périphérique et les pseudopodes sont marqués de façon plus intense. L'identification des mêmes zones est obtenue en microscopie électronique par immunomarquage à l'or colloïdal, ce résultat confirme l'identité des microfilaments dans ces régions (Brugerolle et al., 1996).
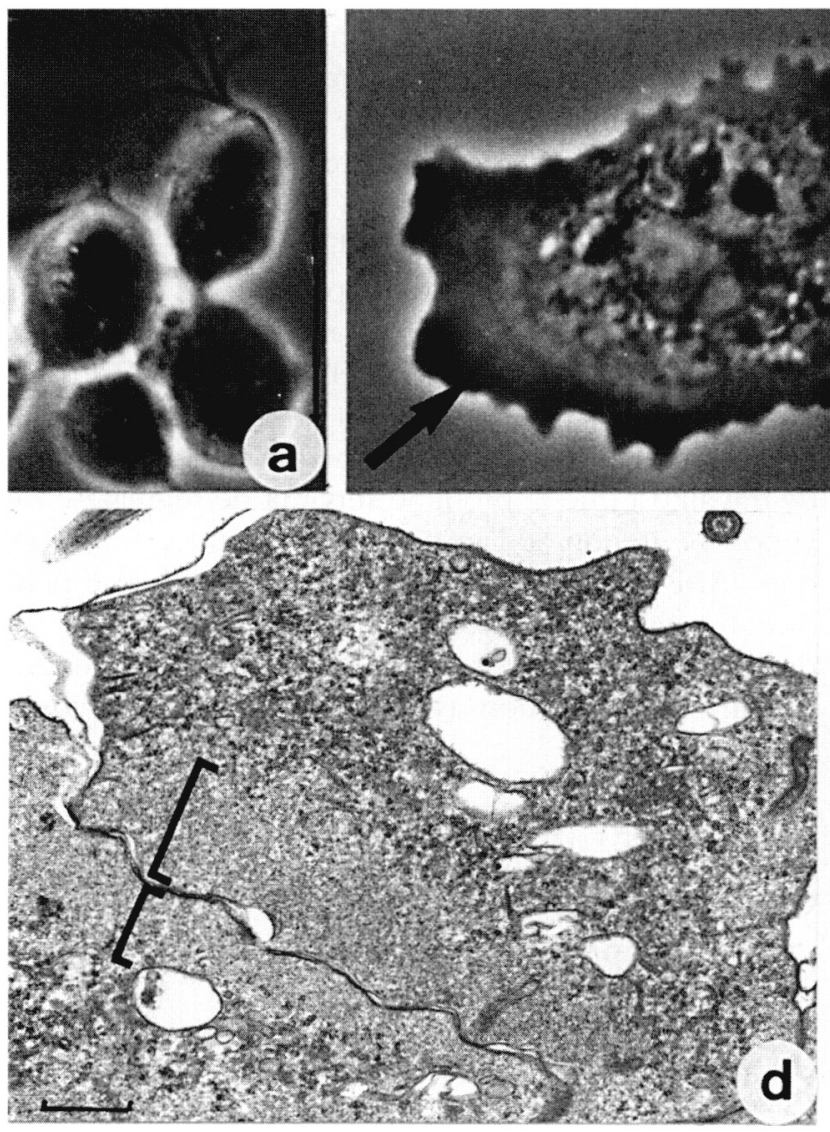
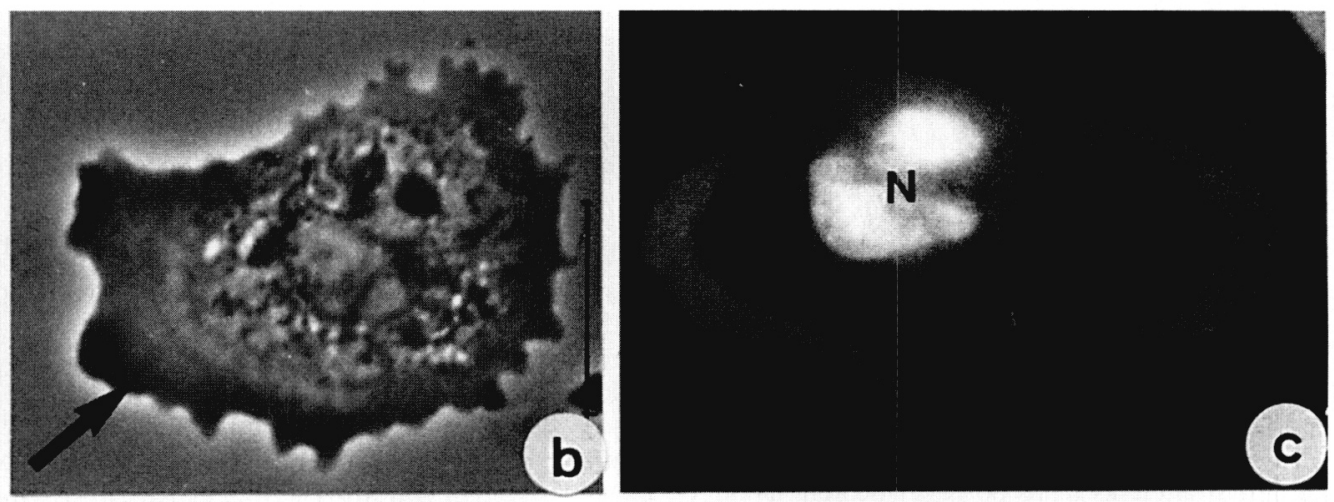

1.

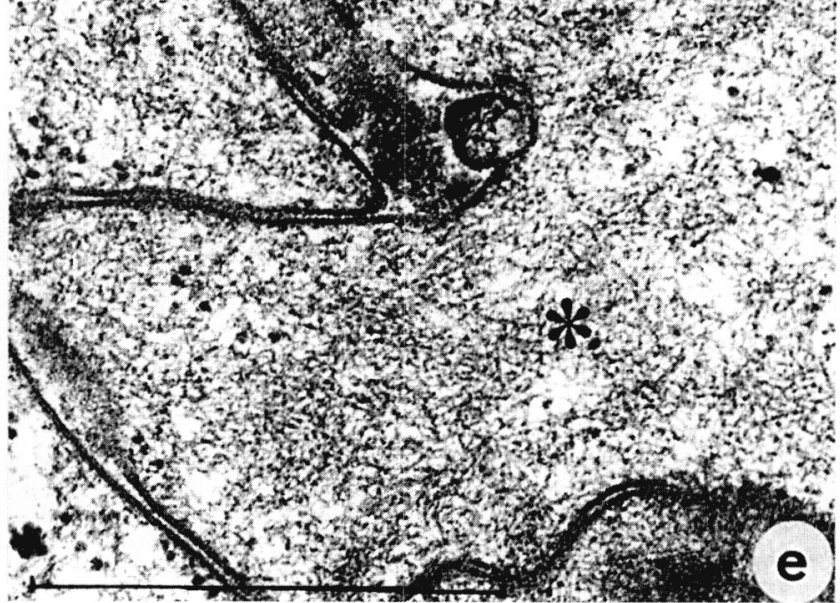

Fig. 2. - Cytosquelette actine de Trichomonas vaginalis.

Forme flagellée ovoïde de Trichomonas vaginalis (barre : $10 \mu \mathrm{m}$ ) (a). Forme amiboïde adhérente à une lamelle de verre, on remarque le cytoplasme homogène dans la frange périphérique et les pseudopodes (flèches) (barre $10 \mu \mathrm{m}$ ) (b). Le marquage par un anticorps antiactine décore la frange périphérique du cytoplasme alors que les noyaux sont marqués au Hoechst 33258 (barre $10 \mu \mathrm{m}$ ) (c). Deux cellules de Trichomonas vaginalis accolées présentent une bande de cytoplasme microfibrillaire dans la zone d'adhérence (D) (barre $1 \mu \mathrm{m})$ (d). Réseau de microfilaments sous-membranaires dans la zone d'adhésion (*) (barre $1 \mu \mathrm{m})$ (e). 
Les gènes codant l'actine ont été recherchés dans une banque de cDNA de T. vaginalis, puis clonés. Par hybridation moléculaire nous avons montré qu'il y a au moins 10 gènes codant cette protéine chez T. vaginalis (Bricheux et Brugerolle, 1997). Le séquençage complet de cinq d'entre eux montre qu'ils codent une protéine de 376 amino acides ayant une masse moléculaire de 41,8 kDa. Les gènes séquencés sont identiques entre eux à $98 \%$; les pourcentages d'identité avec les séquences d'autres organismes varient de 65 à $70 \%$ et les pourcentages de similarité de 80 à $85 \%$. Les plus grandes différences sont trouvées avec l'actine de Giardia, $58 \%$ d'identité et $76 \%$ de similarité. Cette comparaison place les Trichomonadines près de la base de l'arbre des Eucaryotes. Les modifications de séquence les plus marquantes sont situées à l'extrémité carboxy-terminale et dans les domaines de liaison à la DNase I (résidus 38-52), ce qui pourrait expliquer la faible inhibition par la DNase I (Cappuccinelli et al., 1987).

Alors que l'utilisation d'anticorps du commerce pour révéler les protéines associées à l'actine est inefficace chez T. vaginalis, la production d'un Ac monoclonal nous a permis de mettre en évidence une protéine de type $\alpha$-actinine. Les images en IF avec cet Ac sont identiques à celles obtenues avec un Ac anti-actine; ces deux protéines co-localisent révélant des amas sousmembranaires à la périphérie des cellules amiboïdes. La protéine marquée par l'Ac en IF a un masse moléculaire voisine de $100 \mathrm{kDa}$ et se résout en deux spots en électrophorèse bidimensionnelle. Le criblage d'une banque d'expression de T. vaginalis avec l'Ac a permis d'isoler un clone portant le gène qui code une protéine ayant de fortes homologies avec des protéines liant l'actine (ABP) et avec l' $\alpha$-actinine. L'analyse de la séquence en amino-acides déduite de cette protéine, montre qu'elle possède les sites de fixation à l'actine dans le domaine amino-terminal, un domaine central composé d'hélices a, et un domaine carboxy-terminal contenant un motif "EF-hand " de liaison du $\mathrm{Ca}^{2+}$. L'hybridation moléculaire avec l'ADN génomique ne révèle qu'un seul gène. Cette protéine pourrait former des ponts entre les microfilaments d'actine et participer à la liaison des microfilaments avec la membrane plasmique.

La réorganisation des filaments d'actine au cours de l'adhésion et l'action inhibitrice de la cytochalasine D dans l'adhésion montre bien qu'il existe une relation entre adhésion et cytosquelette d'actine. Une signalisation impliquant des protéines membranaires de type adhésines (Alderete et al., 1995) avec des protéines de liaison aux microfilaments d'actine et/ou avec des protéines membranaires est recherchée. Cette signalisation pourrait jouer un rôle dans la régulation du cytosquelette d'actine de T. vaginalis.

\section{PROTÉINES ASSOCIÉES À L'ACTINE CHEZ LES MÉROZOÏTES INVASIFS DE PLASMODIUM}

L

e protozoaire parasite du genre Plasmodium est responsable du paludisme qui sévit essentiellement dans les zones tropicales, où les conditions écologiques favorisent le développement des populations vectrices de moustiques. Le parasite envahit les hématies après s'être différencié en mérozoïte. Des observations morphologiques ont révélé que suivant une interaction initiale entre le mérozoïte et sa cellule hôte, le parasite s'oriente de façon à apposer son extrémité apicale à la membrane plasmique érythrocytaire. Le point de contact évolue progressivement en deux jonctions latérales " mobiles". Les organelles apicaux de sécrétion déchargent alors en partie leur contenu protéo-lipidique et il se produit, conjointement, une invagination de la membrane plasmique érythrocytaire à partir des jonctions. Cette invagination évolue en vacuole parasitophore au sein de laquelle le mérozoïte se propulse activement. Alors que des données récentes ont permis d'identifier les molécules ligands et réceptrices impliquées dans l'interaction précédant la formation de la vacuole chez différentes espèces de Plasmodium (pour une revue, voir Ward et al., 1994), peu d'éléments concernant la nature et la fonction de molécules effectrices contrôlant la réorientation et la pénétration du parasite dans sa vacuole sont actuellement disponibles.

La force motrice exercée par le parasite au moment de son entrée dans la cellule hôte repose sur une réorganisation de son cytosquelette d'actine, en particulier d'un processus de polymérisation de l'actine. Ceci a été suggéré par l'inhibition de l'entrée de mérozoïtes observée lorsqu'ils sont traités par la toxine fongique cytochalasine (Miller et al., 1987) ou par la latrunculine, issue de spongiaires (Ward et Tardieux, résultats non publiés). Une démonstration définitive du rôle de la polymérisation de l'actine parasitaire a été récemment apportée par Dobrowolski et Sibley (1996) lors du processus d'entrée d'un parasite phylogénétiquement apparenté appartenant au genre Toxoplasma et dont la séquence d'invasion revêt des modalités similaires à celles décrites pour le mérozoïte de Plasmodium.

L'actine est codée par deux gènes dans le génome de Plasmodium falciparum; un seul étant exprimé au stade asexué du mérozoïte (Wesseling, 1988). L'actine a pu être détectée sur blot mais la visualisation in situ de filaments ou de monomères n'a pas été rapportée. Dans tous les systèmes étudiés à ce jour, l'assemblage polarisé de monomères d'actine sous forme de filaments et l'organisation spatiale de ces derniers sont 
régulés par une cohorte de protéines associées à l'actine, elles-mêmes étroitement régulées (Stossel, 1993). Afin de comprendre la dynamique de l'actine chez Plasmodium, nous avons cherché à isoler des protéines associées à l'actine et à caractériser leur fonction sur la dynamique de l'actine. La purification de protéines associées à l'actine par chromatographie d'affinité a été le support de notre démarche expérimentale car cette approche s'est déjà révélée fructueuse dans divers systèmes.

Ainsi, avons-nous isolé, à partir de formes invasives de mérozoïtes de Plasmodium knowlesi, les premières molécules parasitaires capables de lier l'actine et de contrôler sa dynamique. Il s'agit d'un complexe de trois protéines qui lie l'actine sous sa forme filamenteuse et qui possède la propriété d'inhiber in vitro la polymérisation d'actine hétérologue. Après marquage métabolique au soufre radioactif de mérozoïtes purifiés à partir de singes Rhésus infectés, les extraits parasitaires ont été chromatographiés sur actine. Parmi les cinq protéines majeures éluées de façon reproductible, une a été identifiée après microséquençage comme une protéine de choc thermique de $70 \mathrm{kDa}$ (HSP/C 70). Son origine parasitaire a été confirmée à l'aide de deux anticorps anti-HSC 70 qui ne présentaient pas de réactivité croisée avec les protéines de stress des hématies. Des expériences de co-immunoprécipation à l'aide d'anticorps dirigés soit contre l'actine soit contre HSC 70 ont renforcé les résultats des protocoles de purification.

Chez l'amibe Dictyostelium discoideum, HSC 70 fait partie d'un complexe de protéines qui contrôle la polymérisation de l'actine observée au cours de la chémotaxie induite par l'AMPc. Les deux autres membres du complexe sont Cap32/34 qui constituent un hétérodimère de $32 \mathrm{kDa}$ et $34 \mathrm{kDa}$ coiffant l'extrémité à croissance rapide des filaments d'actine et contrôlant ainsi la polymérisation (Haus et al., 1993). De telles protéines hétérodimériques sont ubiquitaires et sont capables de réguler la polymérisation de l'actine in vivo (Hug et al., 1995). In vitro, leur activité est indépendante de la concentration en calcium et régulée par les phospho-inositols (fig. 3).

Chez Plasmodium, nous avons co-purifié HSC 70 avec un dimère d'environ $32-34 \mathrm{kDa}$ par chromatographie d'affinité et de gel filtration. Ce complexe inhibe la polymérisation d'actine et co-sédimente avec l'actine filamenteuse. Cette inhibition corrèle significativement avec la concentration en hétérodimère et n'est pas dépendante de la concentration en HSC 70 et en calcium. En revanche, l'effet d'inhibition est annihilé par le phospho-inositol 4.5 biphosphate, lipide cible pour l'activité phospholipase $\mathrm{C}$ impliquée dans la genèse de différents seconds messagers.

Il est clair qu'une identification des protéines $32-34 \mathrm{kDa}$ de Plasmodium par leur séquence nucléotidique res-

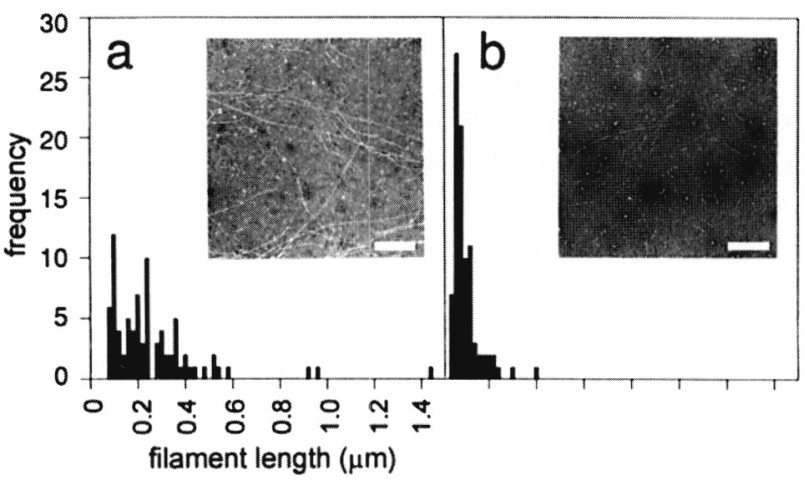

Fig. 3. - Microscopie électronique des filaments d'actine polymérisés en présence et en absence des protéines HSP70 et 32-34 kDa. L'actine a été polymérisée en absence (a) et en présence (b) de la fraction éluée d'une colonne d'ATP-agarose. Après réaction les filaments sont visualisés en microscopie électronique et la longueur de chaque filament a été déterminée. Des champs représentatifs de chaque traitement sont montrés dans l'insertion visuelle; Barre $=0,2$ $\mathrm{mm}$. Le traitement statistique montre une différence significative entre les deux distributions $(\mathrm{P}<0.001)$

pective serait maintenant souhaitable. L'obtention d'anticorps homologues permettrait également de suivre le devenir de ces protéines, notamment au cours du processus invasif puisque les anticorps hétérologues disponibles n'ont pu être utiles. Comment le complexe HSC70-32-34 pourrait-il être impliqué lors de la séquence conduisant à la propulsion du parasite dans sa vacuole? Nous avons évalué qu'environ un tiers de l'actine est sous forme filamenteuse chez le mérozoïte de $P$. knowlesi. Puisque la cytochalasine agit en partie comme une protéine coiffante, il est raisonnable de poser l'hypothèse que la polymérisation de l'actine parasitaire au moment de l'invasion résulte d'un décoiffage de l'extrémité barbée des filaments pré-existants, laissant des extrémités libres pour une élongation.

\section{ACTINE CYTOSQUELETTIQUE ET PROTÉINES ASSOCIÉES CHEZ LES DINOFLAGELLÉS}

L

es Dinoflagellés sont des protistes présentant une très grande diversité. On connaît plus de 4000 espèces qui se répartissent dans les eaux douces et marines. Leur caractéristique essentielle commune réside dans leur structure nucléaire originale et plus particulièrement dans leur processus de division nommé dinomitose (Chatton, 1920).

La division est initiée par le dédoublement des deux bases flagellaires et les chromosomes condensés en permanence se dupliquent par un processus de répli- 
cation encore inconnu. La membrane nucléaire, persistante au cours du cycle, s'invagine et donne naissance à des canaux cytoplasmiques dans lesquels s'établit le fuseau mitotique dont les microtubules voisinent avec diverses protéines. Aux pôles du fuseau mitotique se trouve une région riche en corps de Golgi appelée zone centrosomienne, dans laquelle la présence de diverses protéines a été récemment démontrée : tubuline, p56 homologue de la cycline B (Barbier et al., 1995), CTR210 (Perret et al., 1991), p72 chaperone (Perret et al., 1995). Il est à noter que, lors de la mitose, la tubuline corticale ne se dépolymérise pas contrairement aux autres Eucaryotes excepté une espèce de Cilié (Perret et al., 1993).

Très peu de travaux ont été faits jusqu'à présent concernant la mise en évidence d'actine chez les Dinoflagellés. Roberts et Roberts (1991) avaient démontré la présence de F-actine par immunofluorescence chez quelques espèces de Dinoflagellés et en particulier chez Gymnodinium sanguineum où elle est localisée autour de l'appareil flagellaire. Ce réseau s'étend vers l'avant de la cellule et autour du domaine nucléaire. Chez une deuxième espèce, Peridinium willei, la F-actine est distribuée également dans le cytoplasme, près du bord de la fente du cingulum. On trouve des réseaux adjacents aux plaques thécales, laissant supposer que l'actine interviendrait dans la disposition de ces plaques pendant le processus de cytodiérèse. Schnepf et al., en 1990, avaient démontré la présence d'actine au niveau du sillon de clivage de Prorocentrum micans ce qui suggérait un rôle important de l'actine dans le processus mitotique.

Plus récemment, nous avons démontré la présence d'actine par voies biochimique et immunocytochimique chez un dinoflagellé autotrophe Prorocentrum micans et un dinoflagellé hétérotrophe Crypthecodinium cohnii (Soyer-Gobillard et al., 1996). Après séparation des protéines par électrophorèse monodimensionnelle et immunotransfert, une bande à $44 \mathrm{kDa}$ a été mise en évidence grâce à un anticorps anti- $\alpha$ actine monoclonal, aussi bien sur des extraits de protéines totales que sur des extraits de protéines nucléaires.

En immunofluorescence sur des coupes à congélation des mêmes espèces et après utilisation de divers anticorps anti- $\alpha$-actine, nous avons pu localiser l'actine au niveau du cortex, au-dessous des plaques thécales et reliée à la région périphérique du noyau par des filaments denses, au moment de la phase G1; la région centrosomienne est également fortement marquée de même qu'à l'intérieur du noyau, le nucléoplasme à proximité des chromosomes et la partie pré-ribosomale du nucléole sont fortement marqués (fig. 4a, b, c, d). Ces observations ont été corroborées en microscopie électronique (fig. 4e).
Sur des cellules en division, l'actine se localise au niveau des régions centrosomiennes, dans les canaux cytoplasmiques qui traversent le noyau. Au moment de la cytodiérèse, le sillon de clivage est fortement marqué par l'anticorps anti- $\alpha$-actine. A l'intérieur du noyau, l'actine est toujours présente dans le nucléoplasme et autour des chromosomes (Soyer-Gobillard et al., 1996).

Afin de pouvoir remplir ses différentes fonctions, l'actine nécessite des partenaires. Nous avons localisé une protéine de type myosine dans différents compartiments de la cellule, en immunofluorescence grâce à un anticorps anti-myosine II de muscle lisse et squelettique et à un anticorps polyclonal anti-myosine II d'Acanthamoeba (Baines et Korn, 1990). Il s'agit de la première détection d'une myosine chez les Dinoflagellés (Ausseil et al., 1997). Cette protéine de type myosine a été localisée au niveau des régions centrosomiennes (sphères archoplasmiques) avec des ancrages au niveau de la partie externe de la membrane nucléaire, ainsi qu'à l'intérieur du noyau.

Au vu de ces observations, certaines fonctions peuvent être suggérées pour l'actine au niveau cytoplasmique mais également au niveau nucléaire. La présence d'actine au niveau du sillon de clivage confirme la participation de cette protéine au moment de la cytodiérèse ainsi que cela est le cas chez les Eucaryotes supérieurs. En ce qui concerne la présence de l'actine au niveau du cortex et de la périphérie du noyau, celle-ci pourrait intervenir dans le maintien de la position du noyau ainsi que dans la disposition des plaques thécales lors de la cytodiérèse. L'actine déterminerait donc entre autres la forme de la cellule. Pour l'actine localisée dans les zones centrosomiennes et dans les canaux cytoplasmiques lors de la division, celle-ci pourrait jouer un rôle dans le positionnement des zones centrosomiennes lors de leur migration et aider à la formation des canaux cytoplasmiques. Enfin, au niveau du noyau, l'actine pourrait participer au transport des chromosomes jusqu'à la membrane nucléaire afin qu'ils puissent s'y fixer et se diviser. Elle pourrait aussi participer au maintien de la forme du noyau.

Il semble donc évident que l'actine, protéine bien représentée chez les Dinoflagellés, en association avec d'autres protéines, participe au processus de la mécanique mitotique et de la cytodiérèse.

\section{CONCLUSION}

es protistes, comme toute cellule eucaryote en
mouvement, présentent un front de migration
généré par une réorganisation rapide du cyto- 
Fig. 4. - Distribution de l'actine chez le Dinoflagellé Crypthecodinium cohnii.

a-f : Immunolocalisation de l'actine chez C. cohnii, en microscopie optique sur cryosections (a-d) et en microscopie électronique $(\mathrm{e}, \mathrm{f})$

a : cellule en phase G1. Les filaments d'actine sont visibles dans la région centrosomienne (flèche) et se prolongent par deux bras enserrant le noyau (N). b : noyau en G1 coloré au DAPI. c : cellules en division. A gauche, cellule en prophase tardive dont le noyau est garni de canaux cytoplasmiques (flèches). Les filaments d'actine sont clairement localisés au niveau de ces canaux (flèches), bien visibles sur la coloration au DAPI de la figure $\mathrm{d}$. A droite, une cellule en télophase dans laquelle les deux cellules-filles présentent un long faisceau de filaments d'actine (flèche longue). Le sillon de clivage (cf) est également marqué par l'anticorps (monoclonal anti-aactine $\mathrm{Ab}$, Boehringer). a-d : $\times 5$ 400. e : Cellule cryofixée (Fast Freeze Fixation) et cryosubstituée à l'acétone puis traitée avec l'anticorps antiaactine (Boehringer). Les grains d'or sont localisés dans le nucléoplasme et au niveau du nucléole où la région granulaire préribosomale (G) est marquée. Les chromosomes ainsi que les NORs et le cristal protéique ne sont jamais marqués. $\times 42500$; Barre $=0,5 \mathrm{~mm}$. $\mathrm{f}$ : Détail de la localisation de l'actine au niveau du nucléoplasme fibrillaire à proximité d'un chromosome (ch) (flèche creuse) $\times 75$ 000; Barre $=0,25 \mathrm{~mm}$. Les détails techniques sont développés dans l'article Soyer-Gobillard et al., 1996.
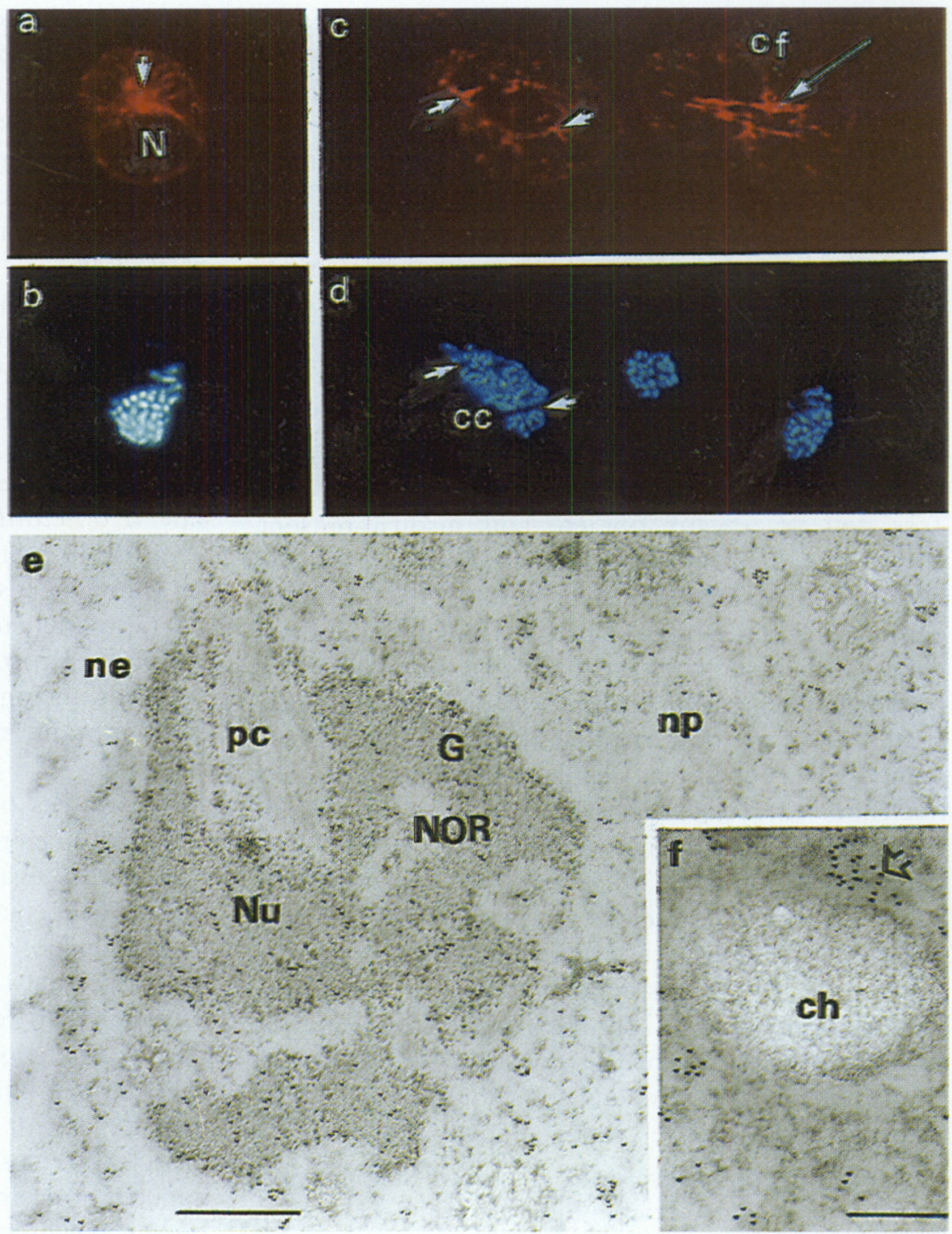

squelette d'actine sous-cortical. Les remaniements du cytosquelette se traduisent par l'émission, au front de migration, d'une projection membranaire riche en actine et dépourvue d'organelles. Les filaments d'actine formés sont orientés avec leur extrémité croissante vers la membrane plasmique, ils sont organisés en réseaux par la participation des protéines telles ABP-120 et $\alpha$ actinine. L'équilibre entre F-actine et G-actine est maintenu par des protéines séquestrantes comme la profiline, des protéines " coiffantes " tel ADF ainsi que le complexe HSP/C70-cap32-34. L'apport des études de biochimie fondamentale ainsi que le développement des techniques moléculaires permettant une analyse génétique pourra nous renseigner in vivo sur l'activité de ces protéines qui lient l'actine. En effet, la possibilité récente de transformer des protozoaires parasites tels Entamoeba, Trichomonas, Plasmodium et Toxo- plasma offre d'énormes perspectives pour l'analyse du rôle du cytosquelette dans la biologie de ces organismes parasites pour l'homme. De plus, des fonctions inattendues sont proposées pour l'actine chez des Dinoflagellés, elle pourrait participer au transport de chromosomes vers la membrane nucléaire lors de la cytodiérèse.

\section{RÉFÉRENCES}

Abe H., Obimata T., Minamide L.S. \& Bamburg J.R. Xenopus laevis actin-depolymerizing factor/cofilin: a phosphorylation-regulated protein essential for development. J. Cell Biol., 1996, 132, 871-885.

Alderete J.F., LehKer M.W. \& ARroyo R. The mechanisms and molecules involved in cytoadherence and pathogenesis of Trichomonas vaginalis. Parasitol. Today, 1995, 11, 70-74. 
Ausseil J., Soyer-Gobillard M.O., Géraud M.L., Baines I., Preston T.M. \& Moreau H. MYO II is an original $75 \mathrm{kDa}$ protein related to cytoskeleton: A biochemical, immunocytochemical and molecular study, 1998, article soumis.

BAINES I.C. \& KORN E.D. Localization of myosin IC and myosin II in Acanthamoeba castelanii by indirect immunofluorescence and immunogold electron microscopy. J. Cell Biol., 1990, 111, 1895-1904.

Barbier M., Albert M., Géraud M.L., Bhaud Y., Picard A. \& SOYer-Gobillard M.O. Cell cycle regulation of the primitive dinoflagellate Crypthecodinium cohnii Biecheler: evidence for the presence of an homolog of cyclin B. Biol. Cell, 1995, 84, 35-42.

Bricheux G. \& Brugerolle G. Molecular cloning of actin genes in Trichomonas vaginalis and phylogeny inferred from actin sequences. FEMS Microbiol. Letters, 1997, 153, 205213

Brugerolle G., Bricheux G. \& Coffe G. Actin cytoskeleton demonstration in Trichomonas vaginalis and in other trichomonads. Biol. Cell, 1996, 88, 29-36.

Cappuccinelli P., Sellito C., Zicconi D. \& Juliano C. Structural and molecular organization of Trichomonas vaginalis cytoskeleton. Acta Universitatis Carolinae Biologica, 1987, 30, 211-217.

Carlier M.F. \& Pantaloni D. Control of actin dynamics in cell motility. J. Mol. Biol., 1997, 269, 459-467.

Chatton E. Les Péridiniens parasites. Morphologie, reproduction, éthologie. Arch.Zool Exp. Gén., 1920, 59, 1-475.

DoBRowolsKi J.M. \& SiBley D. Toxoplasma invasion of mammalian cells is powered by the actin cytoskeleton of the parasite. Cell, 1996, 84, 933-939.

FechHeimer H. \& Zigmond S.H. Focusing on unpolymerized actin. J. Cell Biol., 1993, 123, 1-5.

ForSCHER P. \& SMITH S.J. Action of Cytochalasins on the organization of actin filaments and microtubules in a neuronal growth cone, J. Cell Biol., 1988, 107, 1505-1516.

GUILLÉN N. Role of signalling and cytoskeletal rearrangements in the pathogenesis of Entamoeba bistolytica. TIM , 1996, 4, 191-196.

Haus U. et al. The heat shock cognate protein from Dictyostelium affects actin polymerization through interaction with the actin-binding protein cap32/34. EMBO J., 1993, 12, 3763-3771.

Hug C., Jay P.Y., Reddy I., McNally J.G., Bridgman P.C., ELSON E.L. \& COOPER J.A. Cooper. Capping protein levels influence actin assembly and cell motility in Dictyostelium. Cell, 1995, 81, 591-600.

Mogilner A. \& Oster G. Cell motility driven by actin polymerization. Bioph. Journal, 1996, 71, 3030-3045.

Noegel A.A., Rapp S., LOTSPeich F., SChleicher M. \& STEWART M. The Dictyostelium gelation factor shares a putative actinbinding site with $\alpha$-actinin and dystrophin and also has a rod domain containing six 100-residue motifs that appear to have a cross-beta conformation. J. Cell Biol., 1989, 109, 607-618.
PANTALONI D. \& CARLIER M.F. How profilin promotes actin filament assembly in the presence of thymosin beta 4. Cell, 1993, 75, 1007-1014.

Perelroizen I., Didry D., Christensen H., Chua N.H. \& CarLiER M. F. Role of nucleotide exchange and hydrolysis in the function of profilin in actin assembly. J. Biol. Chem, 1996, 271, 12302-12309.

Perret E., Albert M., Bordes N., Bornens M. \& SoyerGobillard M.O. Microtubular spindle and centrosome structures during the cell cycle in a Dinoflagellate Crypthecodinium cohnii B. an immunocytochemical study. BioSystems, 1991, 25, 53-65.

Perret E., Davoust J., Albert M., Besseau L. \& SoyerGobillard M.O. Microtubule organization during the cell cycle of the primitive eukaryote dinoflagellate Crypthecodinium cohnii. J. Cell Sci., 1993, 104, 639-651.

Perret E., Moudjou M., Géraud M.L., Derancourt J., SoyerGoBillaRd M.O. \& BORNENS M. Identification of an HSP70related protein associated with the centrosome from dinoflagellate to human cells. J. Cell Sci., 1995, 108, 711-725.

RoBerts \& RoBerTs. In: The cytoskeleton of flagellates and ciliates protists. Springer Verlag, Wien, New-york, 1991, 105-122.

Schnepf E., Winter S., Storck I. \& Quader H. A complementary experimental study of cell division in the dinoflagellate Prorocentrum micans. Eur. J. Protistol., 1990, 25, 234-242.

Soyer-Gobillard M.O., Ausseil J. \& Géraud M.L. Nuclear and cytoplasmic actin in Dinoflagellates. Biol. Cell, 1996, 87, 17-35.

STOSSEL T. P. On the crawling of animal cells. Science, 1993, 260, 1086-1094.

VARGas M., SAnsonetTi P. \& Guillén N. Isolation and characterization of the gelation factor ABP-120 from Entamoeba histolytica. Mol. Microb., 1996, 22, 849-857.

Vargas M., Voigt H., Sansonetti P. \& Guillén N. Molecular identification of Myosin IB from the lower eukaryote Entamoeba histolytica, a human parasite. Mol. Biochem. Parasitol., 1997, 86, 61-73.

WANG Y.-L. Exchange of actin subunits at the leading edge of living fibroblasts. J. Cell Biol., 1985, 101, 597-602.

WARD G.E. , ChITNIS C.E. \& MilLER L.H. Invasion of Erythrocytes by Malarial Merozoites, in : Strategies for Intracellular Survival of Microbes. D. Russell (eds), W.B. Saunders Co., London, 1994, 155-190.

Wesseling J.G., de Ree J.M., Ponnudurai T., Smits M.A. \& Schoenmakers G. Nucleotide sequence and deduced amino acid sequence of a Plasmodium falciparum actin gene. Mol. Biochem. Parasitol., 1988, 7, 313-320.

Reçu le 11 décembre 1997 Accepté le 2 avril 1998 